ELOHI

Peuples indigènes et environnement

5-6 | 2014

Ressources du vivant

\title{
Un pays doit-il exploiter toutes ses ressources? Une réflexion sur le cas de l'Équateur
}

\section{Aline Rouhaud}

\section{(2) OpenEdition}

1 Journals

\section{Édition électronique}

URL : http://journals.openedition.org/elohi/779

DOI : 10.4000/elohi.779

ISSN : 2268-5243

\section{Éditeur}

Presses universitaires de Bordeaux

\section{Édition imprimée}

Date de publication : 1 janvier 2014

Pagination : 213-230

ISBN : 979-10-300-0021-4

ISSN : 2437-8175

\section{Référence électronique}

Aline Rouhaud, « Un pays doit-il exploiter toutes ses ressources? Une réflexion sur le cas de l'Équateur », ELOHI [En ligne], 5-6 | 2014, mis en ligne le 01 janvier 2015, consulté le 01 mai 2019. URL : http:// journals.openedition.org/elohi/779; DOI : 10.4000/elohi.779 


\section{Un pays doit-il exploiter toutes ses ressources? Une réflexion sur le cas de l'Équateur}

En 2007, l'Équateur se fit remarquer sur la scène internationale : le président Rafael Correa, élu en 2006, présenta à l'ONU le "projet Yasuní » appelé aussi «Initiative ITT ». La même année, au mois de novembre, son pays réintégra l'OPEP, qu'il avait quittée en 1992, et le président y fit la même proposition : laisser dormir, dans le sous-sol du Parc National Yasuní, un gisement de pétrole contre une compensation financière au manque à gagner.

Huit pays d'Amérique du Sud se partagent l'immense forêt amazonienne de 7 millions de $\mathrm{km}^{2}$, un écosystème complexe qui rassemble la plus grande partie de la forêt tropicale existant sur la planète, ainsi qu'un grand nombre d'espèces animales et végétales. On sait, également, que même si elle n'est pas vraiment "le poumon de la planète ", selon la vision ingénue des années 1970 (Uztarroz) $)^{1}$, elle est en revanche un élément indispensable pour réguler le régime pluviométrique de la planète et son déboisement est un facteur du dérèglement climatique. L’Amazonie est devenue, ainsi, une zone internationale, et ceci pour deux raisons principales : elle représente un patrimoine écologique mondial et, bien souvent, son sort s'est réglé et continue de se régler ailleurs. En effet, la demande de matières premières du marché mondial, la construction de routes et de barrages hydroélectriques, la priorité donnée à lélevage et, donc, au déboisement, afin de ravitailler la planète en viande, le triomphe du modèle consumériste occidental, ont gravement endommagé cette forêt depuis la fin du XIX ${ }^{e}$ siècle.

1. Les références entre parenthèses renvoient aux ouvrages et articles cités dans la bibliographie. Elles y sont classées par ordre alphabétique. 
La proposition du président équatorien de renoncer à exploiter un gisement de pétrole, ressource énergétique convoitée dont on nous annonce régulièrement l'épuisement, surprit la communauté internationale par sa nouveauté, son audace, son sens de l'égalité et de la coresponsabilité, sa vision globale du problème. De plus, l'image médiatique d'un président jeune, populaire, de gauche, écologiste, qui défend, comme le défunt président vénézuélien Chávez, «le socialisme du XXI ${ }^{\mathrm{e}}$ siècle ", contribua à rendre encore plus séduisante son initiative.

Ce projet pose, une fois de plus, la question des rapports difficiles entre économie mondialisée et écologie dans les pays du Sud, et il lance au monde le défi d'un changement de paradigme. Un pays doit-il exploiter toutes ses ressources? Faut-il abandonner l'objectif de la croissance et du développement ? Le projet Yasuní était-il viable et pourquoi fut-il abandonné en août 2013 ? Quel bilan peut-on dresser au niveau international ?

Il s'agira d'expliciter, en premier lieu, les liens qui unissaient l'Initiative ITT et la Constitution équatorienne de 2008, en particulier les passages concernant les droits de la Nature. Puis, de montrer comment ce projet prétendait répondre aux préoccupations de la communauté internationale à propos du réchauffement climatique et pourquoi il fut abandonné. Enfin, je voudrais mettre en relief le rôle joué par la population équatorienne, en particulier les groupes indigènes, dans les tentatives de résolution d'une question globale comme celle du climat.

\section{Naissance de I'« Initiative ITT »}

Quelles circonstances ont présidé à l'apparition de l'« Initiative ITT » en Équateur ? Le sigle "ITT », c'est-à-dire Ishpingo, Tanbococha, Tiputini, correspond à trois champs pétroliers. Ils abritent une réserve estimée à 846 millions de barils. Cette réserve, située dans le Parc National Yasuní, créé en 1979, est classée depuis 1989 par l'Unesco Réserve de la Biosphère. Située à $300 \mathrm{~km}$ à l'est de Quito, elle renferme une des plus grandes biodiversités au monde. Par exemple, un seul hectare du Parc contient 644 espèces d'arbres (Patriarca). Ce parc est également habité par différents groupes ethniques, les Huaorani, Shuar, Kichwa, et une zone déclarée « intangible » abrite des tribus en isolement volontaire, les Tagaeri et les Taromenane (Campaña Amazonía por la vida). Le projet consistait à renoncer à $50 \%$ des recettes pour éviter le rejet dans l'atmosphère de plus de 400 millions de tonnes de gaz carbonique. La compensation demandée était de 3,6 milliards de dollars. Un fonds fiduciaire fut mis en place par le PNUD en 2010 qui devait gérer les dons. Le but était d’aller vers un modèle énergétique différent. L'utilisation de ce fonds, capital et intérêts, devait servir à développer une transition vers les énergies vertes et à créer un centre scientifique pour exploiter les capacités de certaines plantes à dissoudre le plastique ou pour déve- 
lopper des projets médicaux innovants, comme l'utilisation d'amphibiens dans la lutte contre le cancer. Les intérêts du capital devaient servir le développement social, l'écotourisme et interdire le déboisement dans certaines zones protégées (Matthieu le Quang, 2010 a). Les premiers pays donateurs furent l'Allemagne (35 millions), l'Italie (35 millions), l'Espagne (6 millions sous forme de remise de dettes publiques), la Turquie, le Japon, La Russie, la Colombie, le Pérou. Puis, à partir de 2011, ce furent plutôt des entreprises privées qui y participèrent, attirées par différents avantages comme l'utilisation du logo, l'affichage, l'amélioration de leur image (Ambassade de l'Équateur en France). En France, le gouvernement n'accepta pas la demande équatorienne de conversion de la dette en apports au projet, mais les régions, Île-de-France, Rhône-Alpes, Limousin, Nord-Pas-de-Calais, les conseils généraux de Meurthe-et-Moselle et des Pyrénées Orientales contribuèrent au projet, intéressés par le thème des transitions énergétiques et les passerelles qui pouvaient exister entre l'Équateur et la France (Patriarca ; Viva Yasuní).

Rafael Correa tentait d'inverser, ainsi, la logique du pollueur-payeur : on payait pour ne pas exploiter une ressource. Il posait, aussi, le problème de la coresponsabilité entre les pays du Nord consommateurs et ceux du Sud producteurs. Les pays du Sud réclament aux pays du Nord le remboursement d'une dette écologique historique : depuis la colonisation, ils se considèrent victimes du pillage de leurs ressources naturelles qui ont servi à bâtir les sociétés industrialisées et modernes du Nord et ont généré toutes sortes de problèmes environnementaux et sociaux (Latouche, 87). Le projet prétendait également être une alternative au marché des permis d'émission de gaz à effet de serre mis en place en 1997 par le Protocole de Kyoto (Kempf et Laronche, 76).

Pour comprendre la portée de cette initiative, il faut rappeler que l'Amazonie équatorienne fut le théâtre de désastres écologiques de grande ampleur : le procès-fleuve contre la compagnie pétrolière Chevron-Texaco, initié en 1993 par 30000 Equatoriens, a duré 20 ans et s'est terminé par une condamnation de la compagnie. Ce «Tchernobyl de l'Amazonie » explique la thèse du moratoire sur le pétrole (Matthieu le Quang, 2010 b). L'idée de laisser le pétrole sous terre est venue de la société civile et des ONG. Il devint le projet-phare du président Correa, et joua en sa faveur pour sa réélection en février 2013. Ce président jeune, métis, est arrivé au pouvoir en 2006, soutenu en particulier par les associations indigènes et les jeunes générations. Situé à gauche de l'échiquier politique, opposé aux États-Unis et au libéralisme économique, il s’est aussi taillé une réputation de président « vert », parfois présentée comme une simple façade (Zibell).

Bien qu'abandonnée en août 2013 (Mena Erazo), l'Initiative ITT mérite qu'on s'y attarde. D'ailleurs, elle reste d'actualité. En effet, elle aurait pu créer un nouveau paradigme et lancer une dynamique à léchelle continentale dans 
la lutte contre le réchauffement climatique, en présentant la solution de la nonexploitation d'une ressource naturelle comme une alternative au marché des émissions. Au lieu de transformer l'air, une ressource universelle, en valeur marchande, le projet ITT rejoignait les droits de La Nature proclamés dans la Constitution de 2008 qui préconisent une vision biocentrée du monde, opposée à une vision anthropocentrée.

\section{L'« Initiative ITT » et les droits de la Nature}

Dans l'anthropocentrisme, la Nature n'est pas considérée comme un être vivant. L'Homme seul est vivant, placé au centre du monde. La Nature lui est périphérique. Il peut l'exploiter pour satisfaire ses besoins (Dufoing, 25-26). Cette conception vient de la philosophie grecque, du judéo-christianisme, du cartésianisme et de sa théorie des animaux machines (Ferry, 59-61). Elle structure la pensée philosophique, juridique, scientifique et économique du monde occidental et, à travers le colonialisme, elle a pénétré l'Asie, l'Afrique, l'Amérique, continents qui furent soumis à la logique du développement et du progrès.

En revanche, dans une vision biocentrée, la Nature est un être vivant au même titre que l'Homme. Elle doit être défendue en soi et pour soi, elle a une valeur intrinsèque qui ne dépend pas des évaluations de l'Homme, qui lui assigne le plus souvent des fonctions instrumentales (Dufoing, 55). Les intérêts humains ne sont pas supérieurs mais égaux à ceux de la Nature. L'Homme forme un tout avec la Nature, c'est un des quatre principes de la philosophie andine, la relacionalidad (Ávila Santamaría, 209-210).

Au Pérou, en Équateur, en plus des rites officiels incas, il existait des croyances populaires animistes. Il y avait un « huaca » ou esprit, dans chaque lieu. Chaque animal avait un esprit protecteur. Les divinités étaient nombreuses : celle du maïs, de la coca, de la pomme de terre et, bien sûr, la Pachamama, la Terremère, à qui on faisait des offrandes à la fin de chaque cycle agricole (Wachtel, 233-238). Pendant la colonisation, les Espagnols pratiquèrent les campagnes " d'extirpation des idolâtries " pour éradiquer ces croyances mais, à la différence des grands empires qui se sont effondrés, ces croyances ont, non seulement perduré, mais se sont même renforcées (Wachtel, 209-211).

Lexpression juridique de la vision biocentrée se retrouve dans la Constitution équatorienne de 2008, constitution pionnière qui proclame les droits de la Nature dès son préambule, et en particulier dans le chapitre 7, des articles 71 à 74. Les droits de la Nature ont été préparés lors des débats pour l'élaboration de la Constitution. De nombreuses personnalités politiques, scientifiques et juridiques y ont participé, comme : Eduardo Gudynas, secrétaire exécutif du CLAES, le Centre Latino-Américain d'Écologie Sociale, et membre de la 
Commission intergouvernementale du changement climatique ; Alberto Acosta, ex-ministre de l'Énergie et des Mines en 2007, Président de l'Assemblée constituante et membre de cette Assemblée d’octobre 2007 à juillet 2008, économiste et enseignant-chercheur à la FLACSO, la Faculté Latino-Américaine des Sciences Sociales ; Esperanza Martínez, biologiste, coordinatrice de la campagne «Amazonie pour la vie »; Joseph $\mathrm{H}$. Vogel, professeur déconomie de l'université de Puerto Rico Río Piedras, spécialiste de la diversité biologique et du changement climatique.

Les auteurs des droits de la Nature en Équateur ne se privent pas, pour étoffer leur argumentation, de démontrer que la vision biocentrée n’est pas l'apanage des peuples indigènes. Des ponts culturels, philosophiques et politiques existent entre le Nord et le Sud. La cosmovision ancestrale précède celle, beaucoup plus récente, de la deep ecology ou écologie radicale. Ils font de nombreuses références à différents courants de pensée développés par : le philosophe français Michel Serres, le scientifique britannique James Lovelock et l'hypothèse Gaia, le philosophe norvégien Arne Naess, considéré comme le fondateur de l'écologie radicale (Zaffaroni, $72-73 ; 86$ ).

Lécologie radicale, un courant de pensée des années 1970, est considérée plus comme un mouvement philosophique que politique (Dufoing, 53). Cependant, elle a et revendique des implications sociopolitiques qui ont fait l'objet de violentes critiques (Ferry, 194-199). Lécologie radicale défend une vision biocentrée du monde, proclame aussi les droits de la Nature, et s'oppose farouchement au cartésianisme, au néolibéralisme et à l'humanisme. Ce discours peut apparaître inquiétant, car il bouleverse complètement la place de l'Homme au sein de l'univers et attaque les fondements de la civilisation occidentale. D’autres groupes, comme les décroissantistes (Serge Latouche) et les éco-socialistes (Michael Löwy), prônent l'abandon du développement, même durable, et la nécessité d'un changement de paradigme, mais sans adhérer pour autant à une vision biocentrée du monde. Lécologie radicale rejoint, ainsi, la cosmovision ancestrale des peuples indigènes. Le juriste argentin Raúl Zaffaroni s'exprime, d'ailleurs, ironiquement à ce propos : " Es como si los niños de Hegel le dijesen al viejo sabio : tonto, ya lo sabíamos antes de que tú llegaras. Nos venciste por bruto pero por bruto tampoco te diste cuenta de lo que hacías. » (Zaffaroni, 136-137). Lauteur suggère, ainsi, qu'après des siècles de colonisation et d'exploitation, le savoir ancestral, longtemps méprisé, prendrait sa revanche.

Dans le Préambule de la Constitution équatorienne, il est écrit : «Celebrando a la naturaleza la Pachamama de la que somos parte y que es vital para nuestra existencia. »

De même, l'article 71 (chapitre VII, Droits de la Nature) stipule : 
La naturaleza o Pachamama, donde se reproduce y realiza la vida, tiene derecho a que se respete integralmente su existencia y el mantenimiento y regeneración de sus ciclos vitales, estructuras, funciones y procesos evolutivos. Toda persona, comunidad, pueblo o nacionalidad podrá exigir a la autoridad pública el cumplimiento de los derechos de la naturaleza.

Le Préambule souligne la vision holistique, caractéristique des peuples indigènes, dans laquelle Homme et Nature forment un tout. Larticle 71 souligne lobligation de respect pour la Nature, synonyme de vie, et répond à l'objection de la « capacité » faite aux droits de la Nature. En effet, qui pourra représenter et défendre les droits d'une forêt, d'une rivière, d'une montagne, si ce n'est l'homme lui-même ? " [...] car c'est toujours pour l'homme qu'il y a du droit [...] et non l'inverse » (Ferry, 206-207). Si cette objection est un obstacle de taille pour les détracteurs des droits de la Nature, elle ne l'est pas pour ses défenseurs qui rapprochent la situation de la Nature de celle des malades mentaux ou des nourrissons dans la justice anthropocentrée. La Nature est donc un être vivant, un sujet de droit doté de personnalité juridique, et la reconnaissance de ce statut posera aussi le problème des droits de propriété (brevets sur les animaux, les plantes). Autre nouveauté : les représentants de la Nature ne se limiteront pas aux victimes de désastres écologiques mais toute personne, groupe ou communauté qui le désire, pourra protester contre la déforestation, l'usage de pesticides, la propriété des animaux et des plantes, etc. (Ávila Santamaría, 195-200). Le devoir de l'État sera d'encourager ces actions (art. 71) : «El Estado incentivará a las personas naturales y jurídicas a los colectivos para que protejan la naturaleza y promoverá el respeto a todos los elementos que forman un ecosistema ». Pour les partisans des droits de la Nature, c'est un grand pas en avant, un immense progrès. Contrairement à ce qu'affirment leurs détracteurs, ce n'est pas un retour au Moyen Âge mais une façon innovante d'agir avec la technologie moderne, en suivant léthique des populations originaires ancestrales. Et les partisans de ces droits de la Nature de rappeler comment l'abolition de l'esclavage, les droits des femmes, la fin de l'apartheid, semblaient impossibles au début.

Il convient de distinguer les droits de la Nature du droit environnemental, largement présent, maintenant, au niveau international. En effet, le terme « environnement » est anthropocentré, car il présente la Nature de façon périphérique. L'environnement doit être sain, c'est un droit social et économique de l'Homme. On ne parle plus, alors, de développement tout court mais de " développement durable ", c'est-à-dire que l'Homme a intégré le souci du caractère non renouvelable de certaines ressources naturelles mais il propose de continuer à se développer en ayant recours aux énergies dites " renouvelables » ou à des mécanismes comme le marché des droits d’émission, et, plus 
généralement, à tout ce qu'on désigne sous le terme de "croissance verte ". Mais, même quand il est considéré comme durable, le développement suppose la rationalité économique et lucrative et ne sort pas de la logique du marché (Löwy, 35-38 et Latouche, 51-68). Les droits de la Nature impliquent la justice écologique, différente elle aussi de la justice environnementale. Cette dernière fonctionne grâce à un système de compensations financières, alors que la justice écologique demande plus que les mécanismes du marché, elle réclame par exemple un nettoyage des zones affectées, des excuses, de la repentance, et l'application du principe de précaution, c'est-à-dire l'interdiction d' exploiter une ressource, surtout si celle-ci est déclarée non-renouvelable, afin de ne pas compromettre la vie des générations futures (Dufoing, 27). Ainsi, il est dit, dans l'article 72 (Constitución 2008, chapitre VII) : « La Naturaleza tiene derecho a la restauración. Esta restauración será independiente de la obligación que tiene el estado y las personas naturales o jurídicas de indemnizar a los individuos y colectivos que dependan de los sistemas naturales afectados ».

\section{Pratiques de non-exploitation des ressources naturelles}

Le projet ITT s'inscrivait dans des pratiques de non-exploitation d'une ressource naturelle. D’un côté, chez les défenseurs du développement, prévaut une vision utilitaire, mercantiliste de la Nature : elle fournit à l'Homme des ressources naturelles qui vont entrer dans le système productif sous forme de matières premières. L'Amérique du Sud, depuis la colonisation, est pourvoyeuse de matières premières et elle s'est insérée comme telle dans l'économie mondiale, après les guerres d'Indépendance, sans changement de modèle économique (Rouquié, 106-107). L'Amazonie est une zone extrêmement fragile, dont dépend le climat de la planète : l'enjeu est donc global. Le Brésil, par exemple, fait partie des cinq pays qui émettent le plus de gaz à effet de serre dans le monde à cause de la déforestation qu'il pratique en Amazonie pour développer lélevage et la culture du soja, destinée à l'alimentation du bétail (BBCmundo.com, 13/05/2008). Le développement induit le productivisme et la consommation effrénée. Il s'agit de faire entrer ces ressources naturelles dans un processus de production qui ne tient pas compte de la diminution du capital naturel associé, afin d'accumuler des richesses, des bénéfices, de produire chaque fois plus, afin de consommer plus. Certains indigènes, comme l'Indien aymara Fernando Huanacuni, dénoncent le fait que le libéralisme, comme le marxisme, sont productivistes et responsables de désastres écologiques (Huanacuni, 16-18).

De l'autre côté, chez les peuples indigènes, au contraire, on prélève de la $\mathrm{Na}$ ture ce qui est nécessaire à la satisfaction des besoins de l'Homme. La vision biocentrée, respectueuse des équilibres naturels, condamne tout excès, abus, 
effet néfaste, toute cruauté. Elle prône la sobriété et la pauvreté assumée. Les constitutions équatorienne (2008) et bolivienne (2009) proclament l'orientation sociale et économique du bien-vivre ou sumak kawsay, ce qui, en France, rejoindrait ce que lécrivain-agriculteur Pierre Rabhi appelle la " sobriété heureuse » (Rabhi, 66). Ainsi, l'exploitation d'une ressource peut être remise en question si elle menace l'équilibre naturel. En Bolivie, Fernando Huanacuni, guide spirituel aymara, émet des doutes sur le bien-fondé de l'exploitation des richesses minières, car elles nont jamais apporté le bonheur aux habitants (Huanacuni, 34-35). Lécrivaine et militante éco-féministe Vandana Shiva, invitée en 2010 à Quito, raconte comment dans son pays, l'Inde, un grand mouvement populaire a protesté, en 2006, contre l'exploitation minière d'une montagne sacrée, foyer d'une tribu ancestrale. Finalement, les populations indiennes ont gagné la bataille juridique et la bauxite est restée sous terre (Shiva, 138-140).

Le respect du droit territorial est un autre élément qui peut limiter l'exploitation d'une ressource. Bien souvent, les ressources naturelles se trouvent au cœur d'un territoire indigène. Le territoire correspond à une superficie de terre qui a été distribuée à des groupes de populations qui en ont fait la demande, parce qu'ils ont des revendications ancestrales sur ces terres. Ce sont les pueblos originarios. Ils jouissent de l'autonomie et ils doivent être consultés quant à l'utilisation des ressources naturelles qui se trouvent précisément sur leurs territoires, en application de la convention 169 de l'OIT du 7 juin 1989. L'exploitation de la ressource naturelle entre, alors, en conflit avec les droits de propriété collective. Elle se heurte, aussi, à la relation particulière que l'indigène entretient avec sa terre, devenue territoire. L'indigène fait corps avec son territoire dans lequel il s'enracine depuis des siècles, bien avant la colonisation. Le territoire lui confère une légitimité et une identité. Il détermine son mode de vie, conditionne son existence. Si on détruit son territoire, il n'y aura pas de lieu semblable pour lui. La plupart des constitutions de pays d'Amérique latine, ainsi que plusieurs instances internationales, ont reconnu aux Indigènes des droits territoriaux, culturels et linguistiques. Cependant, bien souvent, les limites de ces territoires se superposent à ceux de Parcs Nationaux, qui renferment à leur tour des ressources naturelles. On peut remarquer que les 17 pays qui concentrent les $2 / 3$ des ressources biologiques de la Terre sont aussi ceux des territoires traditionnels indigènes (GLOOBAL). La gestion des ressources naturelles de ces territoires est devenue une source permanente d'affrontements entre, d'une part, l'État ou les compagnies privées, et d'autre part, les populations indigènes. En effet, l'État reste propriétaire des richesses du sous-sol depuis l'époque coloniale, et peut attribuer des concessions de blocs pétroliers à des compagnies privées ou publiques. L'État équatorien se retrouve ainsi dans une situation ingérable : il doit, à la fois, défendre les droits de la Nature, respecter ceux des Indigènes, leur 
droit à être informés et consultés, imposer la justice écologique, et en même temps, il associe, depuis toujours, la lutte contre la pauvreté à l'exploitation des ressources minières et au développement. Cette ambiguïté se reflète dans deux articles de la Constitution :

Se prohibe la actividad extractiva de recursos no renovables en las áreas protegidas y en zonas declaradas intangibles [...] Excepcionalmente dichos recursos se podrán explotar a petición fundamentada de la Presidencia de la República y previa declaratoria de interés nacional por parte de la asamblea nacional que, de estimarlo conveniente, podrá convocar a consulta popular. » (Art. 407,Título VII, Capítulo segundo, Sección tercera, 180);

Toda decisión o autorización estatal que pueda afectar al ambiente deberá ser consultada a la comunidad[...] (Art. 398, Título VII, Capítulo segundo, Sección primera, 177).

\section{Pourquoi l'Initiative ITT fut-elle abandonnée ?}

On comprend que les présidents de la Bolivie, de l'Équateur, du Pérou, quelle que soit leur orientation idéologique, pratiquent tous un capitalisme d'état ou privé et sacrifient au culte du développement. Ils s'inscrivent dans une longue lignée de dirigeants qui prônent la domination de la Nature par l'Homme. Par exemple, en 1812, le Libertador Simón Bolívar disait, après le tremblement de terre de Caracas : "Si la Naturaleza se opone, lucharemos contra ella y haremos que nos obedezca ». En novembre 2009, lors de l'une de ses allocutions du samedi, le président Correa s'exprimait ainsi : «Si la Naturaleza se opone a la revolución ciudadana, lucharemos y juntos la venceremos ». Face à la proposition de ne pas étendre l'exploitation pétrolière en Amazonie bolivienne, le président Morales déclarait : « ¿De qué entonces va a vivir Bolivia si algunas ONG dicen América sin petróleo ? ». Au Pérou, en 2009, l’ex-président Alán García Pérez qualifiait le refus de l'exploitation pétrolière en Amazonie de manière suivante : "Eso es un error gravísimo... " (citations empruntées à Acosta, 331-333). Les préoccupations environnementales ou les droits de La Nature, même inscrits dans la Constitution, viennent au second plan.

Le 15 août 2013, le président Correa annonça que l'Initiative ITT était abandonnée, car les fonds réunis nétaient pas suffisants. Il expliqua sa décision de deux façons différentes : il souligna, avec insistance, le manque de solidarité internationale, et il fit jouer l'argument économique classique, sortir le pays de la pauvreté (BBCmundo.com, 16/08/2013). Pour Rafael Correa, le développement de l'Équateur reste lié à l'extraction du pétrole. Il affirma, pour rassurer ses opposants, que celle-ci n'aurait quasiment pas d'impact sur la Nature grâce à une technologie avancée (Núñez). Pourtant, l'Amazonie reste une des régions 
les plus pauvres du pays et l'exploitation pétrolière, qui représente $20 \%$ du PIB de l'Équateur, n'y a rien changé, bien au contraire (Acosta, 2000). Les bénéfices vont en priorité aux compagnies. Les compagnies pétrolières exploitent l'Amazonie depuis les années 1970. Mais, depuis les années 2000, elles essaient de limiter leur impact environnemental, elles se proclament " propres et efficaces » et cherchent à s'attirer les populations locales. Par exemple, l'espagnole Repsol YPF procédait au reboisement des superficies déboisées, fournissant ainsi du travail aux populations locales. Ou bien construisait des maisons modernes pour les populations qui vivaient à côté des exploitations pétrolières, parfois des dispensaires (Seitz). Par ailleurs, depuis la XI Ronda Petrolera, initiée fin 2012, (convocation des investisseurs pour leur attribuer des concessions de blocs pétroliers), ces compagnies doivent réaliser un investissement minimum obligatoire dans des projets de développement économique et social pour les populations locales. Quant à l'État, il joue le rôle d'intermédiaire entre les compagnies et les populations locales pour gérer cet investissement en fonction des projets demandés (Bellani, 2013). Entre la protection du territoire et l'attrait de la modernité, les groupes indigènes sont divisés : certains approuvent la présence de ces compagnies car ils veulent tirer un maximum d'avantages des Blancs, mais d'autres pointent du doigt les nuisances, les maladies et la fin du mode de vie traditionnel. L'impact est indéniable. La compagnie publique Petroecuador, par exemple, a été responsable de 850 fuites de pétrole depuis 2000, par manque de ressources pour moderniser les oléoducs. Un seul kilomètre de route réduit de 100 hectares la biodiversité. Enfin, en changeant le mode de vie des populations locales, les compagnies créent une culture de la dépendance (Seitz et Lanchín).

Pour le président Correa, l'abandon de l'Initiative ITT était, surtout, léchec de la communauté internationale, et non le sien. Pourtant, cette initiative avait éveillé de nombreux espoirs, en particulier chez les jeunes générations et les communautés indigènes. En 2010, le vice-président Lenin Moreno préconisait même l'emploi d'un nouveau mot, yasunizar, soit le fait de protéger la Terre sacrée (Fernández). En France, le projet avait aussi suscité de l'espoir et de l'enthousiasme (Matthieu le Quang, 2012 et Laetitia Moreau, 2011). L'Équateur semblait ouvrir la voie à d'autres pays dans des conditions similaires, comme le Guatemala ou la Bolivie, où se trouve le Parc National Madidi, de $19000 \mathrm{~km}^{2}$, qui abrite la plus grande richesse biologique de Bolivie, mais aussi de grandes réserves de pétrole d’après le président Morales (Fernández, 2010). Enclencher une dynamique régionale semblait possible. Ce petit pays d'Amérique latine semblait donner une leçon au monde entier : il préférait protéger la biodiversité et les populations indigènes à l'extraction de la ressource pétrolière. Il avait la force de laisser un trésor sous terre. 
Mais le président équatorien devint vite la cible de nombreuses critiques, nationales et internationales (Parienté). On lui reprocha surtout son incohérence, son hypocrisie, un manque de crédibilité, ainsi qu'une mauvaise gestion (Escobar). En effet, au cas où il ne récolterait pas les fonds demandés, le président avait prévu un plan $\mathrm{B}$ qui donnerait le feu vert à l'exploitation du pétrole (Rojas). À plusieurs reprises (mars 2012, février 2013), il lança des appels d'offres aux investisseurs étrangers pour réaliser des concessions de nouveaux blocs pétroliers, ce qui compromettait gravement la crédibilité du projet (Machado). Le président fut, alors, accusé de "chantage écologique » (Parienté). En outre, on fit remarquer qu'il ne respectait même pas les propres lois qu'il avait contribué à inscrire dans la Constitution, lesquelles constituaient déjà une très bonne raison de ne pas exploiter cette zone (Pirard et Billé). Les communautés indigènes, quant à elles, réclamaient un plan $\mathrm{C}$ qui arrêterait l'exploitation du pétrole dans le Parc Yasuní sans demander un dollar (Machado). D’un point de vue technique, le projet apparaissait, finalement, inefficace et difficile à mettre en pratique : il valait mieux jouer sur la réduction de la demande que sur celle de l'offre. Car la conservation du gisement se ferait en contrepartie d'un autre gisement qui aurait été exploité à sa place pour satisfaire la demande. Le gisement en question, d'ailleurs, ne représentait que dix jours de consommation mondiale. Il aurait fallu aussi répliquer l'initiative à grande échelle pour qu'elle soit efficace, ce qui posait le problème de son coût exponentiel (Pirard et Billé).

\section{Le rôle des populations indigènes}

Depuis les années 1990, les désastres engendrés par le développement et le progrès mettent la planète en péril et l'écologie devient un enjeu planétaire. Le danger des gaz à effet de serre sur le climat est la nouvelle menace globale. Il y a consensus sur la nécessité de protéger la planète, mais pas sur les moyens ni sur les échéances. Les Conférences et les Protocoles s'enchaînent sans quémergent vraiment des solutions ou des engagements. De mon point de vue, la question écologique est une des plus difficiles que l'Homme ait à résoudre, car elle ne passe que par des solutions globales et elle implique surtout d'avoir une vision à long terme, ce qui est souvent incompatible avec les politiques et les intérêts nationaux, les rivalités, la concurrence et l'accaparement des richesses.

En revanche, en Amazonie, il y a coïncidence des luttes. L'Indigène, en se mobilisant pour défendre son territoire, lutte en même temps pour la défense de la planète. Ainsi, en Équateur, depuis 1980, la puissante CONAIE, (Confédération des Nationalités Indigènes de l'Équateur), qui rassemble 14 nationalités, a toujours joué un rôle politique et culturel important dans l'histoire du pays. Elle rassemble les peuples andins, ceux de la côte et ceux de l'Amazonie 
(environ 30 \% de ses représentants réunis dans la CONFENIAE). En 1996 est apparu le mouvement Pachakutik, son bras politique, qui lui permet délargir son audience en établissant une connexion entre le mouvement indigène et les non Indigènes (altermondialistes, étudiants, divers groupes de gauche) lors dévènements mondiaux comme le Sommet de Seattle en 1999 ou nationaux, comme l'opposition à la dollarisation de l'économie équatorienne en 2000. Depuis toujours opposés à l'économie extractiviste en Amazonie, (pétrole et mines), et au néolibéralisme, la CONAIE et Pachakutik ont engrangé les succès : droits territoriaux, éducation bilingue (GLOOBAL). Ils ont aussi contribué au renversement ou à l'élection de différents présidents. D’abord favorables au président Correa, qui promettait de «mettre fin à la longue nuit du néolibéralisme ", fermait la base américaine de Manta, lançait la Revolución ciudadana, ils s'en sont progressivement séparés, à partir de 2009, pour renouer avec la tradition historique des marches de protestation. Les affrontements furent parfois violents. Les installations pétrolières en Amazonie, les mégaprojets d'infrastructure du président, la répression des manifestants, furent autant de motifs de déception et de rupture (Denvir). L'abandon du projet ITT en août 2013, n'a fait qu'augmenter les tensions et a souligné, une fois de plus, le divorce entre le président et les jeunes, en particulier.

Le collectif « Yasunidos » est né en février 2014, il regroupe des étudiants, des artistes, des activistes écologistes, des Indigènes huaorani, tous opposés au feu vert donné à l'extraction du pétrole dans cette zone (Paúl Mena Erazo). Le collectif réclame une consultation populaire sur le sujet, mais les signatures pour obtenir un référendum ont été jugées insuffisantes car la plupart d'entre elles ont été invalidées (BBCmundo.com, 6/05/2014). La voie légale semble, donc, épuisée mais il est encore trop tôt pour se prononcer.

Quant à la CONAIE et à Pachakutik, ils sont affaiblis par des divisions internes : une partie a fait alliance avec le gouvernement de Correa, une autre continue de s'y opposer. Certains groupes, (CONFENIAE, La Unidad Plurinacional de las Izquierdas, "Amazonía por la vida ») continuent de réclamer l'abandon de l'exploitation pétrolière dans le Yasuní, alors même que la compagnie Petroamazonas va commencer à opérer dans le bloc ITT (Ecuavisa.com). Ils accusent le président Correa de violer l'article 57 de la Constitution, lequel interdit l'extraction pétrolière dans la zone intangible réservée aux peuples nocontactados (en isolement volontaire). Mais le gouvernement nie la présence de ces peuples dans le périmètre concerné. La CONFENIAE rappelle qu'il y a quarante ans, le peuple Tetete fut exterminé par l'ouverture de l'activité pétrolière dans le Nord (Eluniverso.com). Au Chili, au Pérou, en Equateur, les Indigènes qui protestent contre les entreprises pétrolières, minières ou d'exploitation du bois, apparaissent de plus en plus, aux yeux des pouvoirs en place, comme des 
«terroristes » du $\mathrm{xxI}^{\mathrm{e}}$ siècle. Le président Correa, plus préoccupé par l'opposition de droite, les qualifie, quant à lui, de " gauche radicale » alors que la protestation sociale est un droit inscrit dans la Constitution.

À travers des associations comme la CONAIE ou des mouvements comme Pachakutik, on voit bien que la cause indigène se place résolument à gauche de léchiquier politique. Elle est antilibérale et milite contre les traités de commerce avec les États-Unis ou l'Europe, les mégaprojets du président Correa, les concessions aux compagnies pétrolières et minières. Elle défend un autre paradigme de développement respectueux de la Nature et de l'Homme (Lanchín) et entraîne ainsi, dans son sillage, d’autres groupes de non-Indigènes. On pourrait conclure qu'en Amazonie les luttes syndicales, antilibérales, sont devenues des luttes pour défendre la Nature.

En effet, il est frappant de constater comme certains leaders marxistes ont évolué de l'idéal révolutionnaire vers la défense de la Terre et de l'indianité. C'est le cas du péruvien Hugo Blanco : à 78 ans, Hugo Blanco est passé de la révolution trotskiste à la défense de la Terre et de la Pachamama. Il affirme : "Ser de izquierdas es defender la tierra hoy ( (Pérez). On peut évoquer, également, le combat de Chico Mendes au Brésil (Löwy, 179-189). Pour eux, l'écologie n'est pas un débat politique ou philosophique, c'est une question vitale, urgente, qui affecte directement leur quotidien, comme le fait remarquer le leader péruvien Hugo Blanco : « [...] Et la population d'Amazonie nest-elle pas totalement écologiste, prête à mourir pour défendre ses forêts contre la déprédation ?" (Löwy, 48). Cette " écologie du pauvre " (Löwy, 47) englobe les révoltes des populations paysannes, urbaines, indigènes, directement touchées par la pollution, le déboisement, les activités industrielles, le manque d'eau.

Ces populations ont-elles la capacité de sémanciper du capitalisme d'état ou privé ? Peuvent-elles imposer leurs choix ? Les droits de la Nature, lorientation du bien-vivre, ne doivent pas apparaître comme des conditions qu'on impose de l'extérieur. L’Amazonie représente $30 \%$ du territoire équatorien alors que les Indigènes ne représentent que $10 \%$ de la population. Ils doivent donc convaincre les non-Indigènes du bien-fondé de leurs causes. Certes, ils disposent de nombreux droits, reconnus par les instances internationales, mais ils peinent à les faire appliquer dans leurs propres pays. La Nature est devenue un espace politisé dans lequel s'affrontent différents acteurs. En défendant leurs droits et leurs territoires, les Indigènes peuvent utiliser le langage de lécologisme, ce qui révèle, parfois, la convergence des luttes et des points de vue, mais peut, aussi, brouiller les pistes et apparaittre comme de la manipulation aux yeux des gouvernants. Les ONG, locales ou étrangères, des associations diverses, l'Usaid (l'Agence des États-Unis pour le Développement et l'Aide non 
militaire), sont fréquemment accusées par le pouvoir en place de manipuler les Indigènes, en Équateur comme en Bolivie. En 2012, le président Correa avait annoncé l'expulsion de l'Usaid, l'accusant de fomenter des partis opposés au sien, mais, pour l'instant, elle reste toujours présente en Équateur, bien que toute coopération ait été gelée (Hoy.com.ec).

La proposition de Rafael Correa fut lancée au monde comme un défi, un pari. Le projet, innovant et attractif, réunissait de nombreux acteurs et couvrait plusieurs problématiques à différentes échelles. Au niveau local, il protégeait les territoires des populations indigènes, respectant ainsi la Constitution et les droits de la Nature. Le projet amorçait une transition nationale énergétique vers une économie qui ne se fonderait plus exclusivement sur le modèle extractiviste, caractéristique des pays andins. Enfin, il avait l'avantage de participer à la lutte contre le changement climatique en suivant une logique inverse de la logique marchande des quotas de pollution.

On comprend qu'il ait soulevé l'engouement, au début. Malheureusement, il n’a pas réussi à créer la dynamique nécessaire pour devenir viable. Aucun pays pétrolier n'a suivi l'Équateur. Demander une compensation financière au manque à gagner était une erreur, à mon avis, car ce mécanisme réintroduisait une logique marchande semblable à celle des quotas de pollution, alors que le projet prétendait s'y opposer. Il aurait fallu inventer un autre mécanisme pour aider l'Équateur. Et agir sur la baisse de la demande semblait plus efficace. Le principal intérêt du projet, à mes yeux, reste son caractère innovant.

Le $\mathrm{XXI}^{\mathrm{e}}$ siècle appelle un changement de paradigme. Nous le savons depuis 1972, grâce au rapport Meadows qui s'interroge sur les limites de la croissance (Kempf et Foucart). Aujourd'hui, les désastres écologiques touchent aussi bien les populations des pays développés que celles des pays émergents, et ces désastres vont aller en augmentant. Un pays ne doit pas exploiter toutes ses ressources, si celles-ci mettent en danger l'équilibre écologique de zones sensibles et menacent la vie de groupes de population. Les Indigènes pourraient jouer le rôle de guides que leur confèrent la légitimité et l'antériorité de leur culture ancestrale. Malgré leurs déclarations, malgré les lois qu'ils savent contourner à leur gré, les dirigeants sud-américains, dans leur ensemble, prennent une voie qui reste celle d'un développement fondé sur l'extractivisme, au nom de la lutte contre la pauvreté et de l'impératif de la croissance. Mais ils ne sont pas les seuls. En effet, comment accepter et faire accepter aux populations que le développement puisse avoir une fin ? Comment se décider à abandonner la consommation effrénée pour la sobriété assumée ? Comment changer de modèle de société ? Vaste tâche qui fait peur au monde entier. "Il s'agit là d'une véritable décolonisation de notre imaginaire et d'une déséconomicisation des esprits nécessaires pour changer vraiment le monde 
avant que le changement du monde ne nous y condamne dans la douleur. » (Latouche, 115).

Au terme de cet article, je voudrais souligner l'apport de la Constitution équatorienne de 2008, ainsi que tous les travaux qui ont contribué à son élaboration. Ils ont démontré que des ponts existent entre les droits de l'Homme et les droits de la Nature, entre la pensée occidentale et la pensée indienne, entre les pays du Nord et ceux du Sud. Il faudrait revoir notre définition du bien-être, changer d'instruments d'analyse, dépasser l'opposition entre la Nature (assimilée à l'état sauvage) et la Culture (synonyme de civilisation occidentale), et celle entre droits de l'Homme et droits de la Nature, pour inventer une nouvelle citoyenneté écologique, à la fois locale et globale. C’est le message de l'Equateur au monde.

\section{Bibliographie}

Ambassade d'Équateur en France. "Initiative Yasuní et le secteur privé ». Disponible sur : http://francia.embajada.gob.ec/?page_id=768, consulté le 21 septembre 2013.

ÁVILA SANTAMARÍA, Ramiro. "El derecho de la naturaleza : fundamentos ", in La Naturaleza con derechos. De La filosofía a la política. Alberto ACOSTA, Esperanza MARTINEZ. Quito, Abya Yala-Universidad Politécnica Salesiana, 2011. 173-239.

ACOSTA, Alberto \& MARTÍNEZ, Esperanza. La Naturaleza con derechos. De la filosofía a la política. Quito, Abya Yala, Universidad Politécnica Salesiana, 2011. con derechos. De la filosofía a la política. 317-369.

ACOSTA, Alberto. "Ecuador postpetrolero », in Amazoniaporlavida.org.es., mars 2000. Disponible sur : www.amazoniaporlavida.org.es/Dejar-el-crudoen-el-subsuelo/ecuador-post-petrolero.html, consulté le 20/09/2013.

BBCmundo.com. «Claves para entender el Amazonas». 13/05/2008, consulté le 20/09/2013.

BBCmundo.com. «El mundo nos ha fallado con Yasuní ». 16/08/2013, consulté le 20/09/2013.

BELLANI, Orsetta. "Nueva ronda de licitaciones petroleras en la Amazonía : Ecuador entre extractivismo y conservativismo ", in Kaosenlared.net, 9/05/2013. Disponible sur : www.kaosenlared.net, consulté le 30/05/2013.

Campaña Amazonía por la vida. 2007. Disponible sur : www.amazoniaportavida.org/.es/Parque../el-parque-nacional-yasuni.html, consulté le 20/09/2013.

Constitución de Ecuador. 2008. Disponible sur : www.asambleanacional.gov. ec/documentos/constitución_de_bolsillo.pdf, consulté le 18/09/2013. 
DENVIR, Daniel. « Ruptura entre los movimientos sociales y el Presidente Correa ", in www.cetri.be, 28/08/2008. Disponible sur : www.cetri.be/spip. php?article799, consulté le 10/05/2014.

DUFOING, Frédéric. Lécologie radicale. CH-Gollion, Infolio, coll. " Illico », 2012.

Ecuavisa.com. "Jorge Herrera, nuevo presidente de la Conaie », 18/05/2014. Disponible sur : www.ecuavisa.com/articulo/noticias/nacional/61844-jorgeherrera-nuevo-presidente-conaie, consulté le 20/05/2014.

Eluniverso.com. "Confeniae anuncia vigilia permanente por explotación del Yasuní ", 21/08/2013. Disponible sur : www.eluniverso.com/noticias/2013/08/21/nota/1323656/confeniae-anuncia-vigilia-permanente-explotacion-yasuni, consulté le 20/05/2014.

ESCOBAR, Ramiro. "Yasuní : prohibido perforar », in El País, 19/02/2011, consulté le 20/09/2013.

FERNÁNDEZ, María Ángeles. "El Plan Yasuní se extiende por América », in Publico.es. 20/09/2010, consulté le 21/09/2013.

FERRY, Luc. Le Nouvel Ordre écologique. Paris, Grasset, 1992.

GLOOBAL. Guía de conocimientos sobre pueblos indígenas. Disponible sur : www.gloobal.net, consulté le 10/09/2013.

Hoy.com.ec. "Ecuador congela cooperación de Usaid tras amenazarla con expulsión ", 16/12/2013. Disponible sur : www.hoy.com.ec, consulté le $18 / 12 / 2013$.

HUANACUNI MAMANI, Fernando. "Buen Vivir/Vivir Bien : filosofía, políticas, estrategias y experiencias regionales andinas ", in CAOI (Coordinadora Andina de Organizaciones Indígenas), Lima, février 2010. Disponible sur : www.reflectiongroup.org/stuff/vivir-bien, consulté le 20/09/2012.

KEMPF, Hervé et Martine LARONCHE. « Faut-il mettre en marché le droit de polluer ? ", in Coup de chaud sur la planète. Paris, Le Monde et E.J.L., 2001, 76-78.

KEMPF, Hervé et Stéphane FOUCART. « La croissance mondiale va s'arrêter ", in Le Monde, 25/05/2012, consulté le 8/09/2013.

LANCHÍN, Mike. «Los indígenas ecuatorianos que se enfrentan a las petroleras ", BBCmundo.com. 11/05/2014, consulté le 30/05/2014.

LATOUCHE, Serge. Survivre au développement. Paris, Mille et une nuits, 2004.

LE QUANG, Matthieu. « Une alternative post-Copenhague : l'Initiative Yasuni ITT en Équateur ", in Le Monde, 12/08/2010 a, consulté le 12/08/2013.

- "Une innovation dans la lutte contre le réchauffement climatique : l'Initiative Yasuní ITT en Équateur », in Mouvements. 9/09/2010 b. Disponible 
sur: www.mouvements.info/une-innovation-dans-la-lutte.html, consulté le 21/09/2013.

- Laissons le pétrole sous terre : l'Initiative ITT en Équateur. Montreuil, Omniscience, coll. « Manufacture des idées », 2012.

LÖWY, Michael. Écosocialisme. L'alternative radicale à la catastrophe écologique capitaliste. Paris, Mille et une nuits, 2011.

MACHADO, Decio. "Tres voces distintas y seis manos unidas en defensa de la iniciativa Yasuní ITT ", in Rebelión.org. 6/9/2012. Disponible sur : http:// deciomachado.blogspot.com.es/2012/09/tres-voces-distintas-y-seis-manos. html, consulté le 2/10/ 2013.

MENA ERAZO, Paúl. "¿Por qué fracasó el proyecto ambiental Yasuní en Ecuador? ", in BBCmundo.com.15/08/2013, consulté le 22/09/2013.

MOREAU, Laetitia. "Une idée simple et révolutionnaire ", in Le blog documentaire, 2011. Disponible sur : https://cinemadocumentaire.wordpress. com/2011/12/21/webreportagewebdocumentaire-des-differences/, consulté le $16 / 09 / 2013$.

NUÑEZ, Rogelio. «Rafael Correa se viste de verde contra Chevron », in Infolatam. 23/09/2013. Disponible sur : www.infolatam.com, consulté le 18/10/2013.

PARIENTÉ, Jonathan. «Équateur : Yasuní ITT ou l'échec de la non-exploitation du pétrole ", in Le Monde. 16/08/2013, consulté le 30/09/2013.

PATRIARCA, Eliane. "L'Équateur laissera dans son sous-sol 85 millions de barils ", in Libération. 23/10/2012, consulté le 30/09/2013.

PÉREZ, Sengo. "Hugo Blanco : la tierra con mayúscula ", in Rebelión.org. 5/06/2013. Disponible sur : http://brecha.com.uy, consulté le 18/08/2013.

PIRARD, Romain, BILLÉ, Raphaël. « Ne pas exploiter le pétrole contre une rente : la fausse bonne idée du projet Yasuní ITT », in Slate.fr. 4 avril 2012. Disponible sur : www.slate.fr/tribune/52279/projet-yasuni-itt-petrole-rente, consulté le 8/10/2013.

RABHI, Pierre. Vers la sobriété heureuse. Arles, Actes Sud, 2010.

ROJAS, Carlos A. "El plan B no cala en los jóvenes ", in Elcomercio.com. 17/08/2013, consulté le 8/10/2013.

ROUQUIÉ, Alain. Amérique latine. Introduction à l'Extrême-Occident. Paris, Le Seuil, 1998.

SEITZ, Max. « ¿Petróleo vs conservación? », in BBCmundo.com. 13/05/2008, consulté le 8/10/2013.

SHIVA,Vandana. " Democracia de la Tierra y los derechos de la Naturaleza », in La Naturaleza con derechos. De la filosofía a la política. 137-173.

UZTARROZ, Ricardo. "La fin d'un mythe : Amazonie, poumon de la planète », in Autrement, Série Monde HS nº 49 (octobre 1990), 184-191. 
Viva Yasuní, 2011. Disponible sur : www.vivayasuni.blogspot.fr./p/lassociation-viva-yasuni_5.html, consulté le 13/09/2013.

WACHTEL, Nathan. La vision des vaincus. Paris, Gallimard, coll. « Folio Histoire $», 1971$.

ZAFFARONI, Eugenio Raúl. «La Pachamama y el humano », in La Naturaleza con derechos. De la filosofía a la política. 25-139.

ZIBELL, Matías. «Rafael Correa, el presidente que no se desgasta », in BBCmundo.com/. 18/02/2013, consulté le 8/10/2013.

Résumé : Le président équatorien Rafael Correa avait proposé en 2007 à l'ONU de laisser intacte une partie de la ressource pétrolière dans le sous-sol du Parc National Yasuní contre une compensation financière internationale en vertu de la coresponsabilité en matière environnementale. C'était la première fois qu'une nation promouvait la non-utilisation d'une ressource naturelle comme un moyen de lutte contre le réchauffement climatique. En inversant ainsi le système en vigueur du pollueurpayeur, l'Équateur semblait suivre une logique en harmonie avec sa Constitution de 2008 qui proclame les droits de la Nature. Ce projet innovant avait d'abord séduit la communauté internationale mais bientôt sa faisabilité fut remise en question et l'initiative apparut aux yeux de certains comme un chantage écologique. À la mi-août 2013, le projet fut abandonné par le président, soulevant de nombreuses protestations et critiques, nationales et internationales, toujours d'actualité.

Mots-clefs : Équateur, droits de la Nature et Constitution de 2008, Projet Yasuní ou Initiative ITT, pétrole, réchauffement climatique, communautés indigènes d'Amazonie.

Resumen : El presidente ecuatoriano Rafael Correa había propuesto en 2007 en la ONU dejar sin tocar una parte del crudo en el subsuelo del Parque Nacional Yasuní contra una compensación financiera internacional en virtud de la corresponsabilidad ambiental. Por primera vez una nación impulsaba la no utilización de un recurso natural como medio de lucha contra el calentamiento global. Al invertir así el sistema en vigor que hace pagar al que contamina, Ecuador parecía seguir una lógica en armonía con su Constitución de 2008 que proclama los derechos de la Naturaleza. Este proyecto innovador había seducido, al principio, a la comunidad internacional, pero pronto la realización del proyecto fue cuestionada y la iniciativa apareció, para algunos, como un chantaje ecológico. A mediados de agosto de 2013, el presidente abandonó el proyecto, lo cual dio lugar a numerosas protestas y críticas, nacionales e internacionales, que siguen siendo de actualidad.

Palabras claves : Ecuador, derechos de la Naturaleza y Constitución de 2008, Proyecto, Yasuní o Iniciativa ITT, petróleo, calentamiento global, comunidades indígenas de Amazonía.

Aline Rouhaud est maître de conférences à l'université Paul Valéry Montpellier III, France. Elle enseigne la civilisation de l'Amérique latine contemporaine et actuelle. Son unité de recherche est I'IRIEC, Institut de Recherche Intersite Études Culturelles, Montpellier III/Toulouse II Le Mirail. Son domaine de recherche comprend deux axes : identités et cultures des exilés et des émigrés hispaniques aux États-Unis, en particulier les Cubains de Floride ; et, plus récemment, la relation des populations indigènes avec le développement.

Aline Rouhaud es profesora titular en la universidad Paul Valéry Montpellier III, Francia. Enseña la civilización de América latina contemporánea y actual. Es miembro del IRIEC, Instituto de Investigación Intersitio de Estudios Culturales, Montpellier III/Toulouse II le Mirail. Su área de investigación comprende dos ejes : identidades y culturas de los exiliados y emigrantes hispanos en Estados-Unidos, en particular los cubanos de Miami, Florida ; y, más recientemente, la relación de las poblaciones indígenas con el desarrollo. 